\title{
Reinforcing 'treat to target' for SpA
}

An international task force has presented updated recommendations for treating spondyloarthritis (SpA) to target. The 2017 recommendations, published in Annals of the Rheumatic Diseases, reflect new evidence and insights gained in the years since treat-to-target recommendations for SpA were first put forward in 2012.

When the 2012 recommendations were developed, data from strategic clinical trials were lacking and the recommendations were therefore based on indirect evidence. The task force behind those recommendations thus proposed an ambitious research agenda and anticipated the need to re-evaluate and update the recommendations within a few years. As many of the research questions outlined in that agenda have since been answered, an update was deemed timely.

The 2017 update provides a set of five overarching principles and 11 treat-to-target recommendations for axial and peripheral SpA, including psoriatic arthritis (PsA). The recommendations emphasize the need for shared decision-making between the patient and physician(s) and reinforce that the target of treatment for $\mathrm{SpA}$ should be clinical remission or inactive disease.

\section{Cᄃ}

The recommendations emphasize the need for shared decision-making between the patient and physician(s)

Notably, whereas the 2012 recommendations were all based on the lowest level of evidence (grade D recommendations according to the Oxford Centre for Evidence-Based Medicine grading system, indicating reliance on expert opinion only), five of the 11 recommendations in the 2017 update are stronger (grade B). This change reflects the incorporation of higher quality evidence than that available in 2012, such as data from the TICOPA trial.
For clinical assessment, the recommendations highlight the Ankylosing Spondylitis Disease Activity Score (ASDAS) for SpA and the Disease Activity Index for Psoriatic Arthritis (DAPSA) or Minimal Disease Activity (MDA) for PsA. However, the task force extensively debated and ultimately could not agree on whether scoring systems for PsA disease activity should evaluate disease domains individually or combine them within one score. This and several other matters, such as the need for more data on the response of particular disease manifestations (such as enthesitis and psoriasis) to different therapies, were identified as areas for further research.

Sarah Onuora

ORIGINAL ARTICLE Smolen, J. S. et al. Treating axial spondyloarthritis and peripheral spondyloarthritis, especially psoriatic arthritis, to target: 2017 update of recommendations by an international task force. Ann. Rheum. Dis. http://dx.doi.org/10.1136/

annrheumdis-2017-211734 (2017) FURTHER READING Sieper, J, \& Poddubnyy, D. New evidence on the management of spondyloarthritis. Nat. Rev. Rheumatol. 12, 282-295 (2016) 